\title{
Rediseño y optimización de una máquina dobladora de barras de acero
}

\author{
Nelson Arzola* ${ }^{\S}$, Andrés Tovar*, Alexander Gómez* \\ "Departamento de Ingeniería Mecánica y Mecatrónica, \\ Universidad Nacional de Colombia, Sede Bogotá \\ §e-mail:narzola@unal.edu.co
}

(Recibido: Noviembre 16 de 2006 - Aceptado: Agosto 20 de 2007)

\section{Resumen}

Se presenta un procedimiento de evaluación del desempeño mecánico del sistema formado por la palanca y el rodillo seguidor de una máquina dobladora de barras de acero. Este procedimiento puede ser empleado con fines de diseño o rediseño orientado a sistemas existentes que requieran de mejoras. Se ilustra el procedimiento para el caso de una dobladora para fabricar ganchos de acero que ha tenido continuas pérdidas de funcionalidad por fracturas por fatiga, picadura del rodillo, consumo de potencia elevado y dimensiones de la barra doblada fuera de especificaciones. Se analiza el comportamiento elastoplástico del material de la barra con el fin de conocer la potencia de doblado requerida. Además, se lleva a cabo la definición de los factores de diseño fundamentales tomando en consideración los distintos mecanismos de falla encontrados en esta máquina. El resultado es un sistema de ecuaciones restrictivas que se resuelve para una función objetivo de capacidad de producción. Finalmente, se presenta el rediseño mejorado de la máquina dobladora con nuevas características funcionales.

Palabras clave: Rediseño, Optimización, Máquina dobladora, Elastoplasticidad, Fatiga.

MECHANICAL ENGINEERING

\section{Retrofit and optimization of a steel bar bending machine}

\begin{abstract}
An approach for the evaluation of the mechanical performance of the bar and roller system of a steel bar bending machine is presented. It can be applied for design or retrofit purposes, with emphasis on the improvement of existent systems. The approach is illustrated for the case of a machine that has continuously lost its functionality due to fracture, surface fatigue, high power consumption and shape dimensions outside of specifications. The elastoplastic behavior of the bar is analyzed to determine the power consumption during the bending process. Moreover, the definition of the main design factors that govern the failure mechanisms in these components is carried out. A system of constraining equations is obtained, which is solved for a target function for the production capacity. Lastly, the enhanced redesign of the machine with new functional characteristics is presented.
\end{abstract}

Keywords: Redesign, Optimization, Bending machine, Elastoplasticity, Fatigue. 


\section{Introducción}

En el proceso de diseño de los equipos conformadores, intervienen una serie de parámetros geométricos y características mecánicas, tanto de los componentes que conforman la máquina como del metal trabajado.

Para realizar la deformación plástica, el metal se somete a una tensión que rebasa su límite elástico pero que es inferior a la resistencia a la rotura. $\mathrm{La}$ deformación residual se puede representar como la suma de la deformación plástica de los monocristales de la estructura cristalina del metal.

El tratamiento por presión de los metales puede ser de varios tipos, en función de la temperatura a la cual se efectúa con relación a la temperatura de recristalización, y al procedimiento mediante el cual se realiza la transformación de la forma. Se considera que el proceso se realiza en frío (aún cuando se emplee un calentamiento significativo) si la temperatura del proceso se encuentra por debajo de la temperatura de recristalización del metal. En el caso que se analiza se trata de un proceso de doblado de barras de acero a temperatura ambiente, por lo cual el material se comporta respondiendo con un endurecimiento durante su deformación. La medida del endurecimiento del metal dependerá de sus propiedades mecánicas iniciales, del grado y velocidad de la deformación y de la temperatura.

El establecimiento de una adecuada velocidad de conformado ayuda a los ingenieros de producto a seleccionar el diseño y las condiciones correctas de conformado, con el fin de aumentar la productividad, disminuir el porcentaje de productos fuera de especificaciones y evitar la pérdida de funcionalidad de las piezas del equipo conformador.

En este trabajo se presenta un procedimiento de diseño y comprobación del sistema formado por la palanca y el rodillo seguidor de una máquina dobladora de barras de acero. El procedimiento es aplicado para rediseñar la máquina dobladora tomada como ejemplo ilustrativo, demostrándose lo inadecuado que resulta el diseño original.

\section{Metodología}

El resultado deseado de cualquier proceso de manufactura es lograr productos con la calidad especificada, al menor costo posible, que satisfagan la necesidad del cliente y en la cantidad adecuada. Para un diseño satisfactorio de la palanca y el rodillo seguidor es necesario determinar el conjunto de factores que garantizan las especificaciones del material conformado, y la durabilidad y fiabilidad de operación de la máquina dobladora. Los parámetros fundamentales de conformado que intervienen en la calidad del proceso y del producto final son (Mielnik, 1991):

- Propiedades elastoplásticas del material.

- Temperatura de conformado.

- Grado de deformación.

- Velocidad de deformación.

- Geometrías (formas, secciones, holguras).

- Coeficientes de fricción.

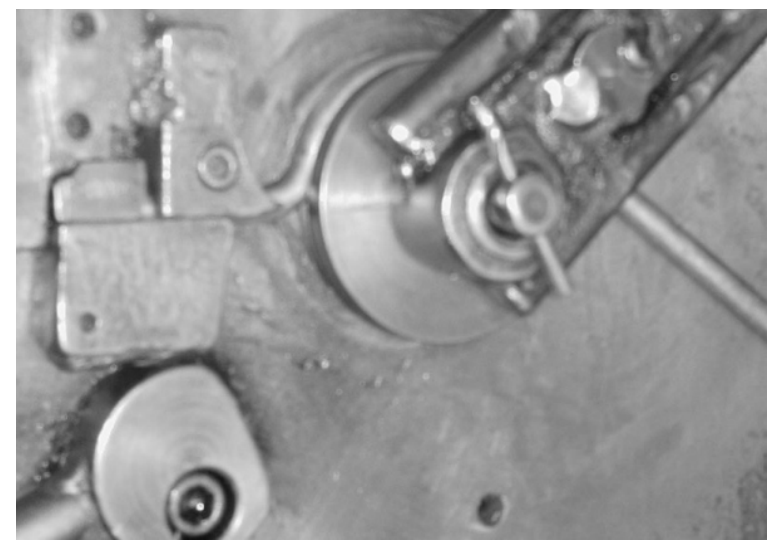

Figura 1. Vista de la máquina dobladora en plena operación.

En el trabajo se analiza el desempeño del sistema formado por la palanca y el rodillo seguidor de una máquina dobladora. Para ello, se emplea como ejemplo ilustrativo, una máquina dobladora de una planta de fabricación de herrajes para baños, la cual, en la Figura 1, se muestra en operación durante el proceso de doblado. 
El historial de funcionamiento de la máquina evidencia numerosas fallas y reparaciones de sus componentes. Durante la inspección visual para analizar las fallas ocurridas, se detectaron fracturas reparadas mediante soldadura y grietas incipientes en la superficie de la palanca del rodillo, generadas por fatiga. En la superficie del rodillo se distinguen hoyuelos que evidencian procesos de picadura y se observan desajustes en las superficies de giro provocados por el desgaste excesivo de los cojinetes. Además, existe insatisfacción debido a las tolerancias muy amplias del producto final, las cuales son generadas fundamentalmente por los desgastes excesivos y por la insuficiente rigidez del mecanismo palanca-rodillo seguidor.

Tomando como premisa que la inspección visual brinda una evidencia empírica irrefutable sobre los puntos de diseño débiles de la solución actual, no se realiza ninguna comprobación sobre su resistencia mecánica, sino que se plantea y resuelve un modelo matemático de optimización que toma en consideración el conjunto de metas de ingeniería que influyen en el correcto desempeño del sistema mecánico objeto de estudio.

Con el objetivo de fortalecer el proceso de rediseño se realiza el despliegue de la función calidad (QFD) para conocer los requerimientos del cliente y trasladarlos a especificaciones de ingeniería. Además, se ejecuta un análisis de modos y efectos de fallas de la máquina actual, con la finalidad de identificar las posibles fallas potenciales y la manera en que pueden ser evitadas (Ullman, 1992).
La formulación matemática utilizada en la solución del problema de optimización propuesto es consistente con la estructura de un problema de optimización no lineal, el cual se puede plantear de manera general (Bhatti, 2000), así :

$$
\begin{aligned}
\min f_{\left(X_{i}\right)} \quad \text { sujeto } a: g_{\left(X_{i}\right)} & \leq 0 \\
h_{\left(X_{i}\right)} & =0
\end{aligned}
$$

Para el caso objeto de análisis, la función objetivo a minimizar es el tiempo de doblado, o lo que es equivalente, se desea maximizar la tasa de producción de los ganchos. Las expresiones restrictivas están dadas por las distintas condiciones de resistencias, sujetas a valores deseados o esperados de los factores de diseño, y otras metas de ingeniería.

Las restricciones para el problema de diseño son las siguientes:

- Potencia consumida $(P)$ en el proceso de doblado.

- Vida útil proyectada para la máquina $\left(T_{\mathrm{v}}\right)$.

- Factor de diseño para la fractura de la barra $\left(\eta_{\mathrm{BE}}\right)$.

- Factor de diseño para la fatiga superficial del rodillo $\left(\eta_{\mathrm{RC}}\right)$.

- Factor de diseño para el aplastamiento de la barra $\left(\eta_{\mathrm{BC}}\right)$.

- Factor de diseño para la deformación angular del eje de giro del rodillo-seguidor $\left(\eta_{\mathrm{DR}}\right)$.

- Factor de diseño para la falla por fatiga de la palanca y los ejes $\left(\eta_{\mathrm{FP}}\right)$.

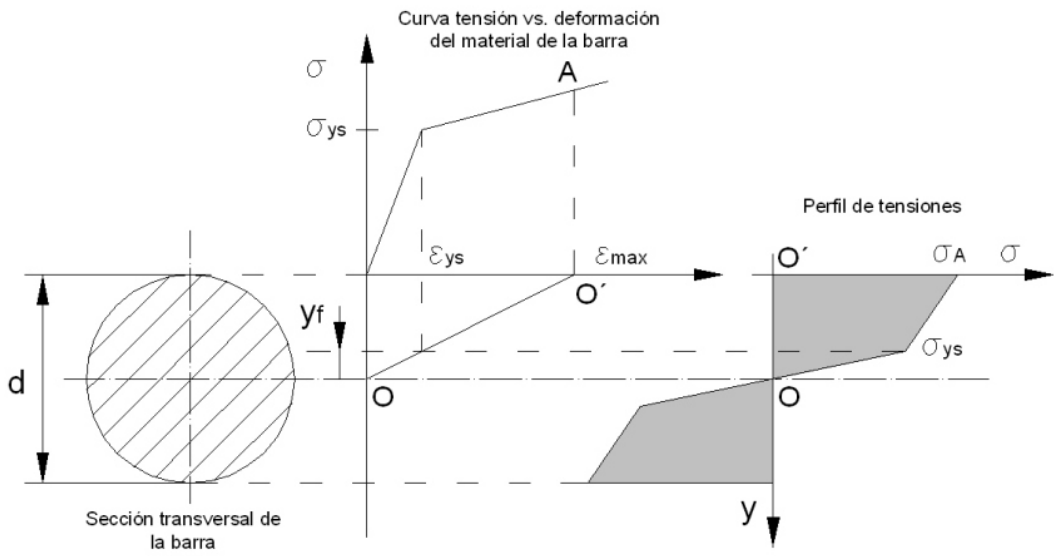

Figura 2. Perfil de tensiones presente en la sección de la barra. 
Las variables de diseño que deberán seleccionarse son las siguientes:

- Dimensiones y geometrías de los distintos elementos de máquina que conforman el sistema doblador.

- Materiales de ingeniería empleados en los elementos de máquina.

A continuación se presenta el desarrollo de las distintas condiciones de resistencia tomadas en consideración.

\subsection{Flexión elastoplástica del material de la barra}

En la Figura 2 se muestra el modo en que se obtiene el perfil de tensiones que surge en la sección transversal de la barra durante su doblado. Para ello, se realiza la proyección de los puntos de la curva de tensión vs. deformación del material, sobre la sección transversal de la barra en el plano de doblado.

El punto O corresponde a la ubicación del eje neutro de la barra y al mismo tiempo al origen de la curva de tensión-deformación, mientras que el punto $\mathrm{O}^{\prime}$ corresponde a la ubicación de la fibra más alejada del eje neutro de la sección transversal de la barra y simultáneamente a la deformación unitaria máxima sobre la curva de tensión vs. deformación (Feodosiev, 1985).

$$
\varepsilon=\frac{y}{R}
$$

donde $\varepsilon(\mathrm{mm} / \mathrm{mm})$ es la deformación normal unitaria , $y(\mathrm{~mm})$ es la posición de la fibra respecto al eje neutro de la sección y $R(\mathrm{~mm})$ es el radio medio de curvatura de la barra. La deformación máxima ocurre en la fibra más alejada respecto al eje neutro:

$$
\varepsilon_{\text {max }}=\frac{d}{2 R}
$$

donde $\varepsilon_{\text {max }}(\mathrm{mm} / \mathrm{mm})$ es la deformación normal unitaria en la fibra más alejada del eje neutro y $d(\mathrm{~mm})$ es el diámetro de la sección transversal de labarra.
El ancho de la sección a la altura $y$ del eje neutro está dada por:

$$
b=2 \sqrt{\left(\frac{d}{2}\right)^{2}-y^{2}}
$$

donde $b(\mathrm{~mm})$ es el ancho de la sección a la altura $y$, respecto al eje neutro.

La sección queda dividida en dos regiones, una sometida a deformaciones elásticas y otra a deformaciones plásticas. La deformación que separa los dos comportamientos en la curva de tensión vs. deformación se obtiene por medio de la siguiente expresión:

$$
\varepsilon_{y s}=\frac{\sigma_{y s}}{E}
$$

donde $\varepsilon_{\text {ys }}(\mathrm{mm} / \mathrm{mm})$ es la deformación normal unitaria para el comienzo del flujo plástico del material de la barra, $\sigma_{\mathrm{ys}}(\mathrm{MPa})$ es el límite de fluencia del material de la barra y $E(\mathrm{MPa})$ es el módulo de elasticidad del material de la barra.

La ordenada que divide las dos regiones en la sección transversal de la barra está dada por:

$$
y_{f}=\frac{\sigma_{y s} R}{E}
$$

donde $y_{\mathrm{f}}(\mathrm{mm})$ es la altura respecto al eje neutro que divide la región elástica y la región plástica en la sección.

El momento flector $M_{\mathrm{f}}(\mathrm{N} \mathrm{mm})$ a aplicar a la barra para que se deforme se relaciona con el perfil de tensiones desarrollado, de acuerdo con:

$M_{f}=\int_{-d / 2}^{d / 2} \sigma b y d y=2 \int_{0}^{y_{f}} \sigma b y d y+2 \int_{y_{f}}^{d / 2} \sigma b y d y$

La curva de tensión vs. deformación del material puede ajustarse por medio de dos funciones lineales. 
Para el rango elástico:

$$
\sigma=E \varepsilon=\frac{E y}{R}
$$

Para el rango plástico:

$$
\sigma=\sigma_{y s}+H\left(\varepsilon-\varepsilon_{y s}\right)=\sigma_{y s}\left(1-\frac{H}{E}\right)+\frac{H y}{R}
$$

donde $H(\mathrm{MPa})$ es la pendiente de la curva tensión vs. deformación en el rango plástico.

La solución de la Ec. (7) es:

$$
\begin{aligned}
M_{f}= & \frac{\sigma_{y s}}{3}\left(1-\frac{H}{E}\right)\left[\left(\frac{d}{2}\right)^{2}-\left(\frac{\sigma_{y s} R}{E}\right)^{2}\right]^{3 / 2} \\
& +\frac{\sigma_{y s} d^{2}}{8}\left(1-\frac{H}{E}\right) \sqrt{\left(\frac{d}{2}\right)^{2}-\left(\frac{\sigma_{y s} R}{E}\right)^{2}} \\
& +\frac{(E-H) d^{4}}{32 R} \operatorname{sen}^{-1}\left(\frac{2 \sigma_{y s} R}{E d}\right)+\frac{\pi H d^{4}}{64 R}
\end{aligned}
$$

La Ec. (10) define la relación que existe entre el momento flector necesario para el doblado con las propiedades elastoplásticas, resistencia mecánica y geometría de la barra.

Un hecho significativo es la dependencia del comportamiento de deformación de la barra con la velocidad de deformación. La curva tensión vs. deformación se vuelve más esbelta para una mayor velocidad de deformación. Este hecho es más acentuado cuando se emplea un proceso de doblado a mayor temperatura. Producto de lo anterior, el consumo de potencia no es una función lineal de la velocidad de doblado.

El esfuerzo sobre la fibra de material, situada a una altura $y$ del eje neutro, depende de la velocidad de deformación de la siguiente manera (Groover, 1997):

$$
\sigma=E^{\prime} \varepsilon^{n} \dot{\varepsilon}^{m}=E^{\prime}\left(\frac{y}{R}\right)^{n}\left(\frac{y \omega}{\theta R}\right)^{m}
$$

donde $\sigma(\mathrm{MPa})$ es la tensión sobre el material de la barra, $E^{\prime}(\mathrm{MPa})$ es el coeficiente de resistencia, $n$ (adimensional) es el exponente de endurecimiento por deformación, $m$ (adimensional) es el exponente de sensibilidad a la velocidad, $\dot{\varepsilon}(\mathrm{mm} / \mathrm{mm} \mathrm{s})$ es la velocidad de deformación, $\omega(\mathrm{rad} / \mathrm{s})$ es la velocidad angular de la palanca del rodillo seguidor y $\theta(\mathrm{rad})$ es el ángulo de giro de la palanca del rodillo seguidor.

La velocidad angular de la palanca es función del volumen de producción a alcanzar por unidad de tiempo. El tiempo de producción se define como la suma de los tiempos de sujeción, doblado y liberación del gancho. La velocidad angular de la palanca se expresa como:

$$
\omega=\frac{\theta C}{3600 \varphi T_{v}}
$$

donde $C$ (unidades producidas) es el volumen de producción proyectado para la vida útil de la máquina, $\varphi=0.7$ es la fracción del tiempo unitario de producción que se destina al proceso de doblado y $T_{\mathrm{v}}(\mathrm{h})$ es la vida útil proyectada para la máquina.

El factor de diseño para la fractura de la barra $\eta_{\mathrm{BE}}$ (adimensional) queda expresado como:

$$
\eta_{B E}=\frac{\sigma_{u t}^{\prime}}{\sigma_{\left(y=\frac{d}{2}\right)}} \geq\left[\eta_{B E}\right]
$$

donde $\sigma_{\text {ut }}^{\prime}(\mathrm{MPa})$ es la resistencia última real del material de la barra y $\left[\eta_{\mathrm{BE}}\right]$ (adimensional) es el factor de diseño permisible para la fractura de la barra.

\subsection{Potencia requerida para el giro del rodillo seguidor}

El proceso de doblado de la barra se realiza forzando a la barra a deformarse de manera elastoplástica contra un dado circular con ayuda de un rodillo seguidor, tal como se muestra en la Figura 3. 


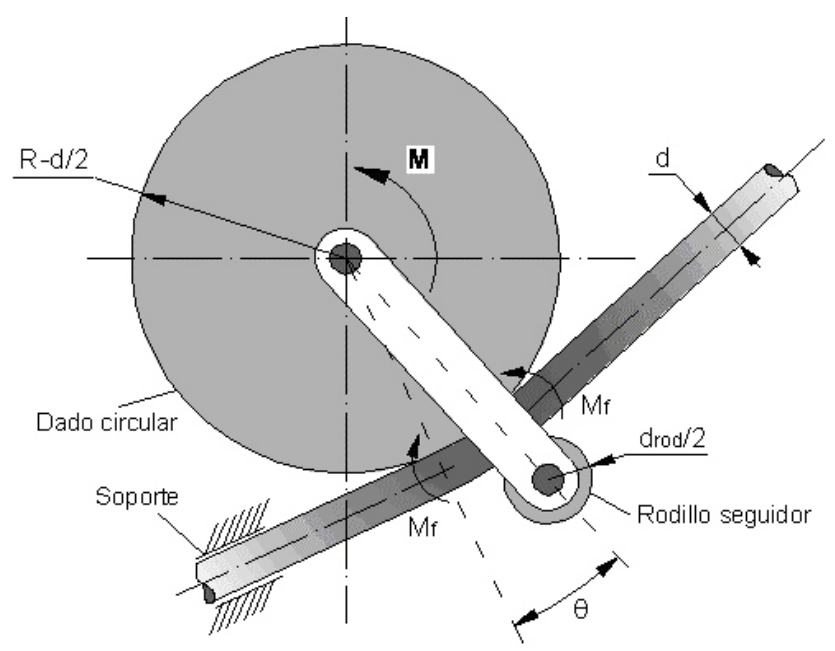

Figura 3. Diagrama del proceso de doblado de la barra.

Para que el proceso de doblado ocurra, el rodillo seguidor deberá adelantarse cierto ángulo respecto al último punto de contacto de la barra con la matriz. De esta forma se provocará un momento flector sobre la sección de la barra, el cual es responsable de la deformación elastoplástica. Por ello, en el diseño de la palanca del rodillo seguidor habrá que garantizar cierta holgura.

En la Figura 4 se representa esta situación. El momento flector existente en el último punto de contacto de la barra con la matriz es:

$$
M_{f}=F \quad b_{f}
$$

donde $F \quad(\mathrm{~N})$ es la fuerza de contacto entre el rodillo seguidor y la barra y $b_{f}(\mathrm{~mm})$ es el brazo de la fuerza de contacto respecto al último punto de contacto entre la barra y la matriz.

La longitud entre centros de la palanca del rodillo seguidor es expresada por:

$$
L=\frac{1}{2}\left(D+2 d+d_{r o d}\right)+\delta
$$

donde $L(\mathrm{~mm})$ es la longitud entre centros de la palanca del rodillo seguidor, $d_{\text {rod }}(\mathrm{mm})$ es el diámetro del rodillo seguidor y $\delta(\mathrm{mm})$ es la holgura requerida para el avance del rodillo seguidor.

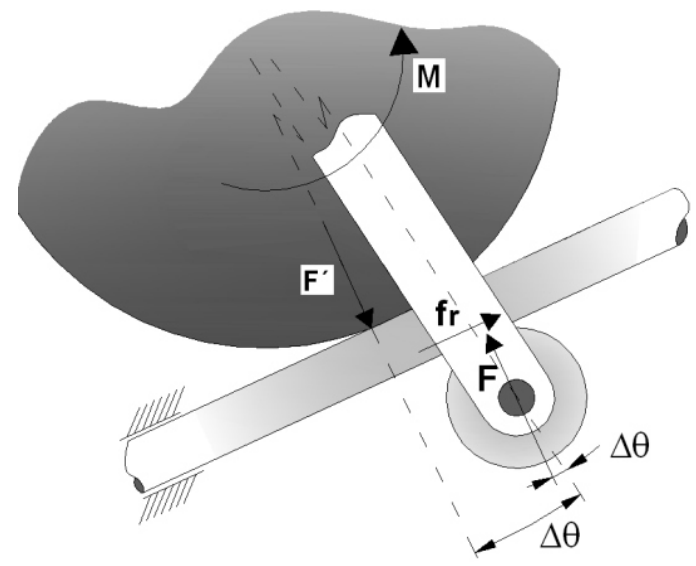

Figura 4. Diagrama de cargas sobre la barra durante el proceso de doblado.

El brazo de momento está dado por:

$$
b_{f}=L \operatorname{sen}(\Delta \theta) \cong L \Delta \theta
$$

donde $\Delta \theta(\mathrm{rad})$ es la posición angular relativa entre las fuerzas de doblado $F$ y $F^{\prime}$.

Las cargas sobre el eje de la palanca son:

$\sum F_{r}=0:$

$O_{r}=F(\cos \Delta \theta-\mu \operatorname{sen} \Delta \theta) \cong F(1-\mu \Delta \theta)$

$\sum F_{t}=0:$

$O_{t}=F(\operatorname{sen} \Delta \theta+\mu \cos \Delta \theta) \cong F(\mu+\Delta \theta)$

$\sum M_{B}=0:$

$M \cong F\left[L(\mu+\Delta \theta)-\mu \frac{d_{r o d}}{2}\left(1+\Delta \theta^{2}\right)\right]$

donde $O_{r}(\mathrm{~N})$ es la carga radial sobre el eje de giro de la palanca, $O_{t}(\mathrm{~N})$ es la carga tangencial sobre el eje de giro de la palanca y $M(\mathrm{~N} \mathrm{~mm})$ es el momento torsor sobre el eje de giro de la palanca. 
El ángulo de avance del rodillo seguidor queda definido por la geometría de la dobladora, el diámetro de la barra y la holgura $\delta$ :

$$
\Delta \theta=\frac{\pi}{2}-\operatorname{sen}^{-1}\left(\frac{D+d+d_{r o d}}{D+d+d_{r o d}+2 \delta}\right)
$$

Finalmente, $M$ queda expresado como:

$$
M=M_{f}\left[1+\frac{\mu}{L \Delta \theta}\left(L-\Delta \theta^{2} \frac{d_{r o d}}{2}-\frac{d_{r o d}}{2}\right)\right]
$$

La potencia requerida para el doblado de la barra está dado por:

$$
P=\frac{10^{-6} K_{S} M \omega}{n_{t}}
$$

donde $P(\mathrm{~kW})$ es la potencia consumida en el doblado, $n_{t}$ (adimensional) es la eficiencia mecánica y $K_{s}=2$ es el factor de servicio.

Una vez doblada la barra y liberada de la acción del rodillo seguidor, las tensiones elásticas restauradoras intentarán descargar la sección transversal de la barra, forzando a la región deformada plásticamente a regresar a su estado inicial.

En la Figura 5 se muestra la superposición de las tensiones de carga y de descarga, y el perfil de tensiones residuales resultantes en la sección de la barra.

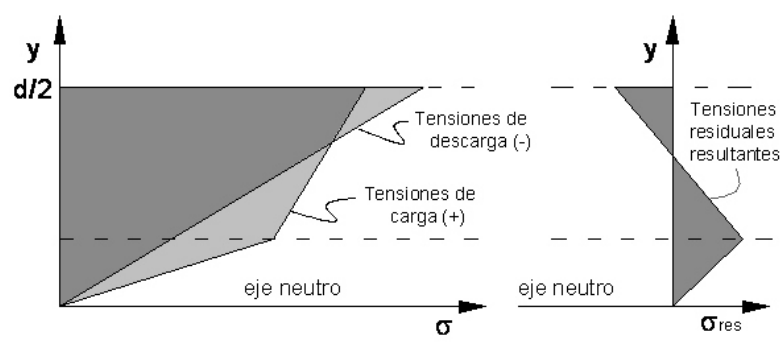

Figura 5. Perfil de tensiones residuales en la sección transversal de labarra.
El efecto visible generado por estas tensiones residuales es el aumento del radio de curvatura de la barra doblada. El radio $\bar{R}_{f}(\mathrm{~mm})$ necesario de la matriz para obtener una barra con un radio medio de doblado final es:

$$
D_{\text {matriz }}=\frac{1}{\frac{1}{2 \bar{R}_{f}}+\frac{32 M_{f}}{\pi E d^{4}}}-d
$$

donde $D_{\text {matriz }}(\mathrm{mm})$ es el diámetro de la matriz y $R_{\mathrm{f}}(\mathrm{mm})$ es el radio medio de doblado final de la barra.

\subsection{Fatiga superficial del rodillo y aplastamiento de la barra}

La capacidad de carga del rodillo está restringida por su resistencia a la fatiga superficial.

La superficie a través de la cual se transmite la carga entre el rodillo y la barra es muy pequeña siendo elevadas las tensiones de contacto que actúan. En este caso el área de contacto es elíptica. La tensión de contacto máxima se expresa como (Norton, 1998):

$$
\sigma_{C, \max }=\frac{3}{2} \frac{F}{\pi L_{1} L_{2}}
$$

donde $\sigma_{C, \text { max }}(\mathrm{MPa})$ es la tensión de contacto máxima y $L_{1}$ y $L_{2}(\mathrm{~mm})$ son los semiejes de la huella de contacto, con:

$$
\begin{aligned}
L_{1} & =k_{L 1} \sqrt[3]{\frac{3 F \Delta}{4 A}} \\
L_{2} & =k_{L 2} \sqrt[3]{\frac{3 F \Delta}{4 A}} \\
\Delta & =\frac{1-V_{r o d}^{2}}{E_{r o d}}+\frac{1-V^{2}}{E}
\end{aligned}
$$


donde $k_{L 1}=f_{(B / A)}, k_{L 2}=f_{(B / A)}$ (adimensionales) son los coeficientes de determinación de las dimensiones de la huella, $\Delta\left(\mathrm{MPa}^{-1}\right)$ es la constante elástica reducida de los materiales del rodillo y la barra, $E_{\text {rod }}(\mathrm{MPa})$ es el módulo de elasticidad del material del rodillo y $v_{\text {rod }}$ y $v$ (adimensionales) son los coeficientes de Poisson del material del rodillo y de la barra respectivamente, con:

$$
\begin{gathered}
A=\frac{0.0476}{d}+\frac{1}{d_{r o d}} \\
B=\left[\frac{1}{d^{2}}+\left(\frac{1}{d_{r o d}}+\frac{1}{1.05 d}\right)^{2}-\frac{2}{d}\left(\frac{1}{d_{r o d}}+\frac{1}{1.05 d}\right)\right]^{1 / 2}
\end{gathered}
$$

La tensión de contacto máxima $\sigma_{C \text {, max }}$ se expresa finalmente como:

$$
\sigma_{C \max }=\frac{3 F^{1 / 3}}{2 \pi k_{L 1} k_{L 2}\left(\frac{3 \Delta}{4 A}\right)}
$$

El factor de diseño para falla por fatiga superficial del rodillo se define por:

$$
\eta_{R C}=\frac{10^{\frac{1}{2 \lambda}\left[\xi-\log \left(\frac{C(D+d) \theta}{2 \pi d_{r o d}}\right)\right]}}{\sigma_{C, \max } \sqrt{\pi \Delta}} \geq\left[\eta_{R C}\right]
$$

donde $\lambda$ (adimensional) es la pendiente de la curva de fatiga superficial $\sigma$ vs. $N$, $\xi$ (adimensional) es el intercepto de la curva de fatiga superficial y $\left[\eta_{\mathrm{RC}}\right]$ (adimensional) es el factor de diseño permisible para falla por fatiga superficial del rodillo.

El factor de diseño para aplastamiento de la barra es:

$$
\eta_{B C}=\frac{\sigma_{C, \lim }}{\sigma_{C, \max }} \geq\left[\eta_{B C}\right]
$$

donde $\sigma_{C \text {, lim }}(\mathrm{MPa})$ es la tensión de contacto límite del material de la barra y $\left[\eta_{\mathrm{BC}}\right]$ (adimensional) es el factor de diseño permisible para aplastamiento de labarra.

Algunas recomendaciones para un diseño correcto del rodillo, orientadas a minimizar los efectos de la picadura, son:

- Emplear superficies altamente pulidas.

- Incrementar la dureza superficial y garantizar un núcleo tenaz.

- Garantizar un diseño rígido para evitar deflexiones significativas y desalineaciones.

- Emplear materiales limpios (o sea, libres de inclusiones).

- Operar con lubricante preferiblemente de viscosidad elevada.

\section{4 Ángulo de deformación del eje de giro del rodillo seguidor}

El ángulo de inclinación del eje del rodillo seguidor es un parámetro de rigidez de la máquina que garantiza que las dimensiones finales de la barra doblada se encuentren dentro de los límites especificados.

Para la determinación del ángulo de deformación del eje de giro del rodillo seguidor son consideradas las deformaciones que se producen solamente en los ejes y en la palanca del rodillo seguidor y son despreciadas las deformaciones producidas en el resto de los componentes de la máquina. Esto es cierto mientras sea suficientemente robusta la estructura de la máquina donde va ensamblada la matriz central y los cojinetes de rodillos cónicos del eje de la palanca. En la Figura 6 se muestran las dimensiones críticas de la palanca del rodillo seguidor.

Las inclinaciones del eje del rodillo provocadas por las cargas de funcionamiento son calculadas empleando el Teorema de Castigliano. Estas inclinaciones se determinan por: 


$$
\begin{gathered}
\gamma_{v}=\frac{F(1-\mu \Delta \theta)}{E}\left\{\frac{32 s_{1}^{2}}{\pi D_{1}^{4}}+\frac{12 s_{1} L}{w^{4}}+\frac{32\left[\left(s_{1}+L\right)^{2}-s_{1}^{2}\right]}{\pi D_{3}^{4}}\right\} \\
\gamma_{h}=\frac{F(\Delta \theta+\mu)}{E}\left\{\frac{32 s_{1}^{2}}{\pi D_{1}^{4}}+\frac{12(L+v) s_{1} L}{w^{4}}+\frac{32\left[\left(s_{1}+s_{3}\right)^{2}-s_{1}^{2}\right]}{\pi D_{3}^{4}}\right\}
\end{gathered}
$$

donde $\gamma_{\mathrm{v}}$ y $\gamma_{\mathrm{h}}$ (rad) son las deformaciones angulares horizontal y vertical del eje del rodillo, respectivamente, $s_{1}$ y $s_{3}(\mathrm{~mm})$ son las longitudes del eje del rodillo y del eje de la palanca, respectivamente, $D_{1}$ y $D_{3}(\mathrm{~mm})$ son los diámetros del eje del rodillo y del eje de la palanca, respectivamente y $w(\mathrm{~mm})$ es el lado de la sección transversal de la palanca.

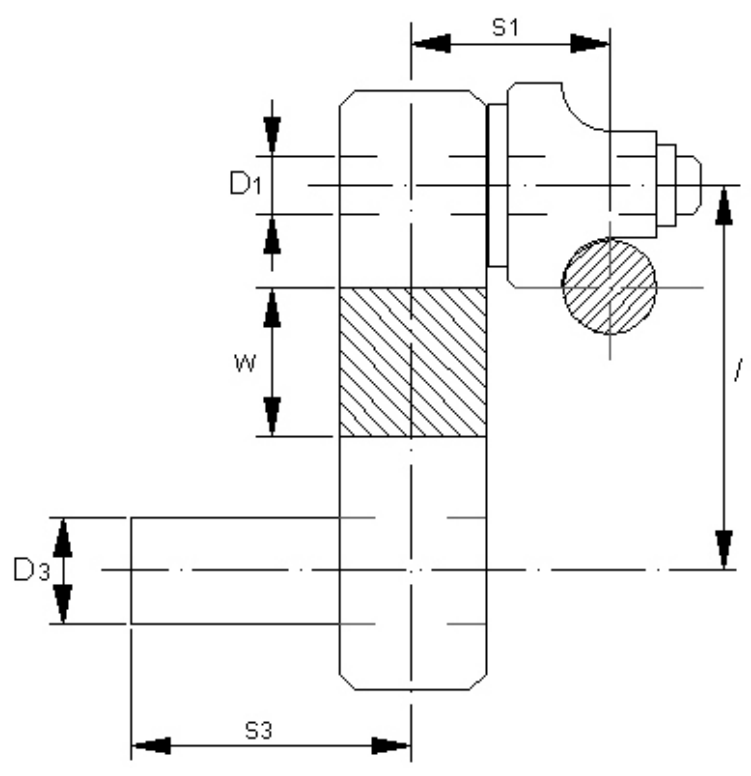

Figura 6. Dimensiones críticas de la palanca-rodillo seguidor.
El factor de diseño para la deformación angular del eje de giro del rodillo seguidor se define como:

$$
\eta_{D R}=\frac{\gamma_{\text {perm }}}{\max \left\{\gamma_{v}, \gamma_{h}\right\}} \geq\left[\eta_{D R}\right]
$$

donde $\gamma_{\text {perm }}$ (rad) es la deformación angular máxima permisible en uno de los planos y $\left[\eta_{D R}\right]$ (adimensional) es el factor de diseño permisible para la deformación angular del eje de giro del rodillo seguidor.

\subsection{Fatiga volumétrica de la palanca y los ejes}

La falla por fatiga volumétrica de la palanca o los ejes podrá presentarse con mayor probabilidad en los puntos donde existan concentraciones de tensiones generadas por la geometría. Por esta razón, se deberán evaluar los ajustes por interferencia de los ejes y el extremo del eje de la palanca donde se encuentra el escalón de apoyo del cojinete de rodillos cónicos derecho.

El estado tensional para estas ubicaciones es multiaxial; debido a esto, se emplea el criterio de falla por fatiga de Von Mises. El factor de diseño para la falla por fatiga de la palanca del rodillo seguidor y de los ejes queda expresado por:

$$
\eta_{F P}=\min \left\{\frac{2 \sigma_{f i} \sigma_{u t}}{\left(\sigma_{f i}+\sigma_{u t}\right) \sigma_{i}}\right\} \geq\left[\eta_{F P}\right]
$$


siendo:

$$
\sigma_{f i}=\left\{\begin{array}{lc}
\sigma_{e i} & \text { para } C>10^{6} \\
\frac{0.81 \sigma_{u t}^{2}}{\sigma_{e i}} C^{-0.33 \log \left(\frac{0.9 \sigma_{u t}}{\sigma_{e i}}\right)} & \text { para } 10^{3} \leq C \leq 10^{6}
\end{array}\right.
$$

$$
\sigma_{e i}=0.484 A_{s} \sigma_{u t}^{1+B_{s}} D_{s, i}^{-0.097}
$$

donde $\sigma_{f i}(\mathrm{MPa})$ es la resistencia a la fatiga para determinado número de ciclos de carga, $\sigma_{e i}(\mathrm{MPa})$ es el límite de resistencia a la fatiga corregido para la ubicación $i, \sigma_{i}(\mathrm{MPa})$ es la tensión efectiva Von Mises máxima para la ubicación $i$ potencialmente peligrosa, $\sigma_{u t}(\mathrm{MPa})$ es la resistencia última a tracción del material de la palanca del rodillo seguidor y los ejes, $\mathrm{A}_{\mathrm{s}} \mathrm{y} \mathrm{B}_{\mathrm{s}}$ (adimensionales) son el coeficiente y el exponente de acabado superficial, respectivamente, $D_{s, i}(\mathrm{~mm})$ es el diámetro equivalente de la sección $i \quad \mathrm{y}\left[\eta_{\mathrm{FP}}\right]$ (adimensional) es el factor de diseño permisible para la falla por fatiga de la palanca del rodillo seguidor y los ejes.

\section{Resultados y discusión}

En la Figura 7 se muestra como varía la relación $M / M_{f}$ para distintos valores de la holgura. En la Tabla 1 se muestran las propiedades del material y la geometría de la barra para la cual se obtuvo el resultado anterior, utilizando como material un acero AISI 1020 laminado en caliente. En la Figura 8 se aprecia como varía la relación $M / M_{f}$ para distintos valores del diámetro relativo del rodillo para una holgura $\delta$ igual a $1 \mathrm{~mm}$. Se demuestra que resulta provechoso emplear una holgura y un diámetro del rodillo seguidor tan grandes como las restricciones geométricas lo permitan. La velocidad de doblado de la barra posee una influencia débil en la respuesta tensión vs. deformación del material. Por ejemplo, un incremento de la velocidad de giro de la palanca del rodillo seguidor desde $0.4 \mathrm{rad} / \mathrm{s}$ hasta 12.4 $\mathrm{rad} / \mathrm{s}$ provocará una elevación del esfuerzo en la fibra más cargada de la barra de solo $3.84 \%$.
Las restricciones de diseño desde el punto de vista de resistencia están definidas por las Ecs. (13), (31), (32), (35) y (36). La vida útil deseada para el conjunto palanca rodillo seguidor se define en cinco años a un turno de trabajo diario y se desea una potencia consumida durante el doblado no mayor a $1 \mathrm{~kW}$. De manera que las restricciones planteadas inicialmente son:

$$
\begin{gathered}
1.2 \leq\left\{\eta_{B E}, \eta_{R C}, \eta_{B C}, \eta_{D R}, \eta_{F P}\right\} \leq 2.0 \\
T_{V} \geq 10400 \mathrm{~h} \\
P \leq 1 \mathrm{~kW}
\end{gathered}
$$

El límite inferior para los factores de diseño garantiza que no ocurra la pérdida de la funcionalidad, mientras que el límite superior garantiza la racionalidad en la cantidad y calidad de los materiales empleados. En la Tabla 2 se muestra el conjunto de variables de diseño empleadas en los cálculos y el valor final alcanzado para cumplir con las metas de diseño requeridas. La función objetivo definida es el volumen de producción generado por la máquina, el cual deberá ser el máximo posible.

Se preseleccionaron un conjunto de materiales de ingeniería que resultaban adecuados en una primera aproximación. Finalmente, el material escogido para el rodillo seguidor y la matriz es un acero AISI 4340 endurecido por inducción hasta una dureza HRC 58, mientras que el material seleccionado para los ejes y la palanca es un acero al carbono AISI 1040 templado y revenido a $200^{\circ} \mathrm{C}$.

No fue posible obtener un factor de diseño para aplastamiento de la barra por contacto con el rodillo superior a la unidad. Sin embargo, esto no es requisito debido a la amplia tolerancia de forma especificada para la sección transversal de la barra.

En la Figura 9 se muestra el rediseño final, el cual cuenta con algunas ventajas adicionales. Por ejemplo, el empleo de un actuador de giro para efectuar el giro de la palanca del rodillo seguidor y una matriz que se desplaza dentro de una camisa para facilitar la salida de la barra doblada. 


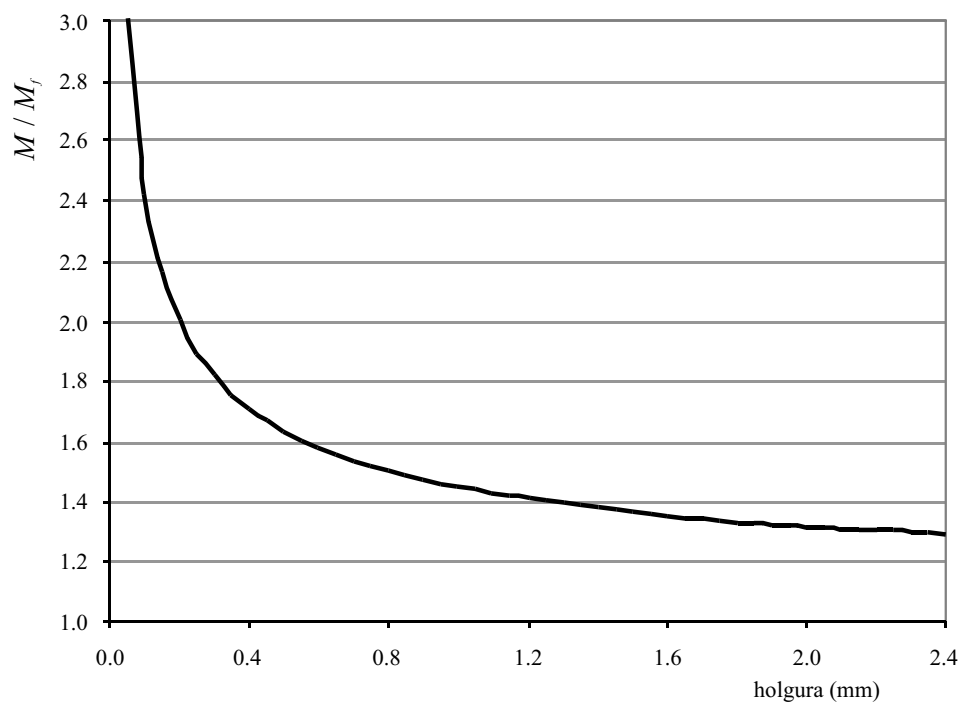

Figura 7. Comportamiento de la relación $M / M_{f}$ con respecto a la holgura del rodillo seguidor.

Tabla 1. Propiedades mecánicas y características geométricas requeridas.

\begin{tabular}{cc}
\hline Propiedades y características & Valor \\
\hline Límite de elasticidad del material $(\mathrm{MPa})$ & 207 \\
\hline Módulo de elasticidad del material $(\mathrm{MPa})$ & 207000 \\
\hline Pendiente en la región de comportamiento plástico $(\mathrm{MPa})$ & 1070 \\
\hline Diámetro de la matriz $(\mathrm{mm})$ & 87.49 \\
\hline Diámetro del rodillo $(\mathrm{mm})$ & 40.0 \\
\hline Diámetro de la barra $(\mathrm{mm})$ & 10.0 \\
\hline Coeficiente de fricción & 0.1 \\
\hline
\end{tabular}

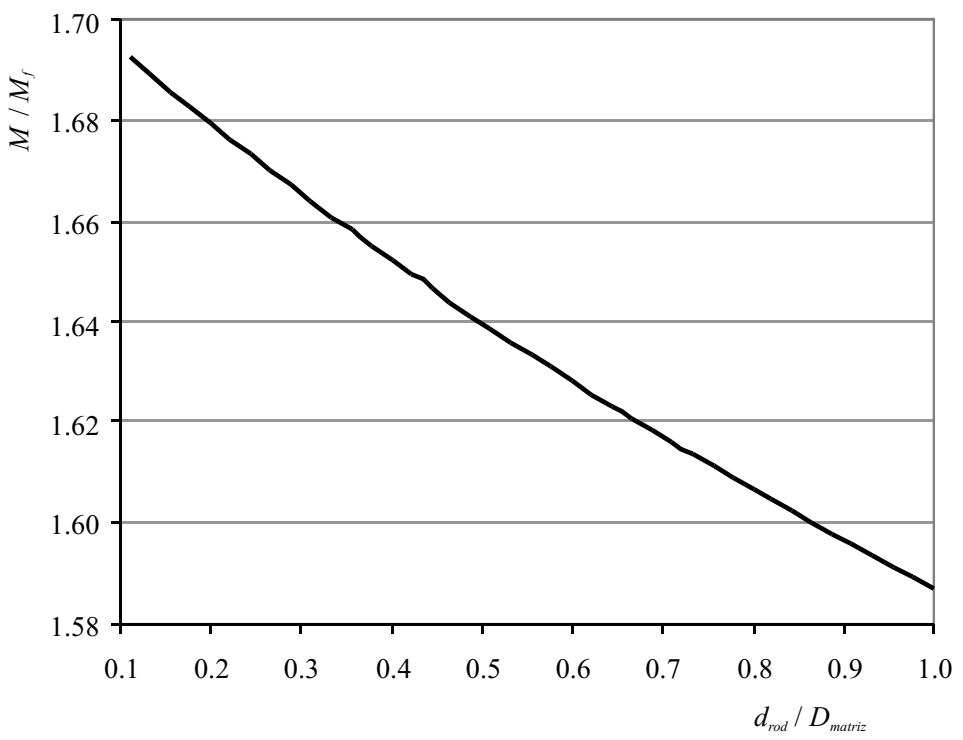

Figura 8. Comportamiento de la relación $M / M_{f}$ con respecto al cociente $d_{\text {rod }} / D_{\text {matriz }}$. 
La incorporación de estas soluciones permite semiautomatizar el proceso de doblado de la barra.

Al realizar una comparación entre el diseño original y el propuesto es posible apreciar cambios significativos: las geometrías son ahora más robustas; los cambios de sección propuestos son realizados con radios de acuerdo mayores; la longitud entre centros de la palanca de doblado es definida en el diseño actual bajo consideraciones adecuadas sobre su influencia en el fenómeno de doblado, mientras que en el diseño original fue establecida bajo un concepto empírico. El material del rodillo y la matriz, originalmente en acero al carbono, es cambiado por un acero de baja aleación endurecido por inducción; y se incluyen algunas ventajas constructivas, no existentes en el diseño original, que facilitan el proceso de sujeción, doblado y liberación de la barra.

Todos estos cambios garantizan un nuevo diseño cualitativamente superior de la máquina dobladora de barras, soportado por un modelo matemático de optimización, que incluye factores de diseño para los modos de pérdidas de funcionalidad o de la condición de operación. La nueva máquina dobladora fabricada muestra excelente resultados en su funcionamiento y desempeño, garantizándole a la empresa indicadores de productividad, calidad y confiabilidad adecuados en el proceso de doblado de las barras.

\section{Conclusiones}

El diseño original del conjunto palanca y rodillo seguidor presenta serias deficiencias, evidenciadas en la inspección visual realizada, que provocan la pérdida de la funcionalidad y vuelven a la máquina dobladora poco confiable. Los resultados de rediseño obtenidos demuestran lo inadecuado de los materiales y geometrías empleados en el diseño original.

El empleo de un sistema de ecuaciones formado por los distintos factores de diseño permite una visión integral del proceso de diseño, fácil de usar y que consume poco tiempo de cálculo.

Existe una influencia débil de la velocidad de deformación en el comportamiento tensión vs. deformación del material de la barra a la temperatura que se realiza el doblado.

Se necesita una potencia de doblado relativamente alta cuando por cuestiones de restricciones geométricas se requiere de una holgura pequeña en la palanca del rodillo seguidor. La potencia de doblado es poco sensible al diámetro relativo del rodillo seguidor, siendo menor para una relación cercana a la unidad.

Tabla 2. Variables de diseño empleadas y el valor final alcanzado para cumplir con las metas de diseño requeridas.

\begin{tabular}{lllc}
\hline \multicolumn{1}{c}{ Variables de diseño } & tipo & valor & unidad \\
\hline Diámetro del eje del rodillo $\left(D_{1}\right)$ & continua & 20.0 & $\mathrm{~mm}$ \\
\hline Diámetro del eje de la palanca $\left(D_{3}\right)$ & continua & 29.0 & $\mathrm{~mm}$ \\
\hline Diámetro del rodillo seguidor $\left(d_{\text {rod }}\right)$ & continua & 40.0 & $\mathrm{~mm}$ \\
\hline Longitud entre centros de la palanca del rodillo seguidor $(L)$ & continua & 74.95 & $\mathrm{~mm}$ \\
\hline Longitud del eje del rodillo $\left(s_{1}\right)$ & continua & 55.0 & $\mathrm{~mm}$ \\
\hline Longitud del eje de la palanca $\left(s_{3}\right)$ & continua & 55.0 & $\mathrm{~mm}$ \\
\hline Lado de la sección transversal de la palanca $(w)$ & continua & 50.0 & $\mathrm{~mm}$ \\
\hline Ángulo de giro de la palanca del rodillo seguidor $(\theta)$ & continua & 5.76 & $\mathrm{rad}$ \\
\hline Velocidad de giro de la palanca del rodillo seguidor $(\omega)$ & continua & 0.952 & $\mathrm{rad} / \mathrm{s}$ \\
\hline Holgura requerida para el avance del rodillo seguidor $(\delta)$ & continua & 1.20 & $\mathrm{~mm}$ \\
\hline Deformación angular permisible en uno de los planos $\left(\gamma_{\max }\right)$ & continua & 0.012 & $\mathrm{rad}$ \\
\hline Factor de servicio $\left(K_{S}\right)$ & continua & 2.0 & $\mathrm{adim}$. \\
\hline Eficiencia mecánica $\left(n_{t}\right)$ & continua & 0.80 & $\mathrm{adim}$. \\
\hline Material para el rodillo seguidor y matriz central & discreta & AISI 4340 & - \\
\hline Material para la palanca y los ejes & discreta & AISI 1040 & - \\
\hline
\end{tabular}




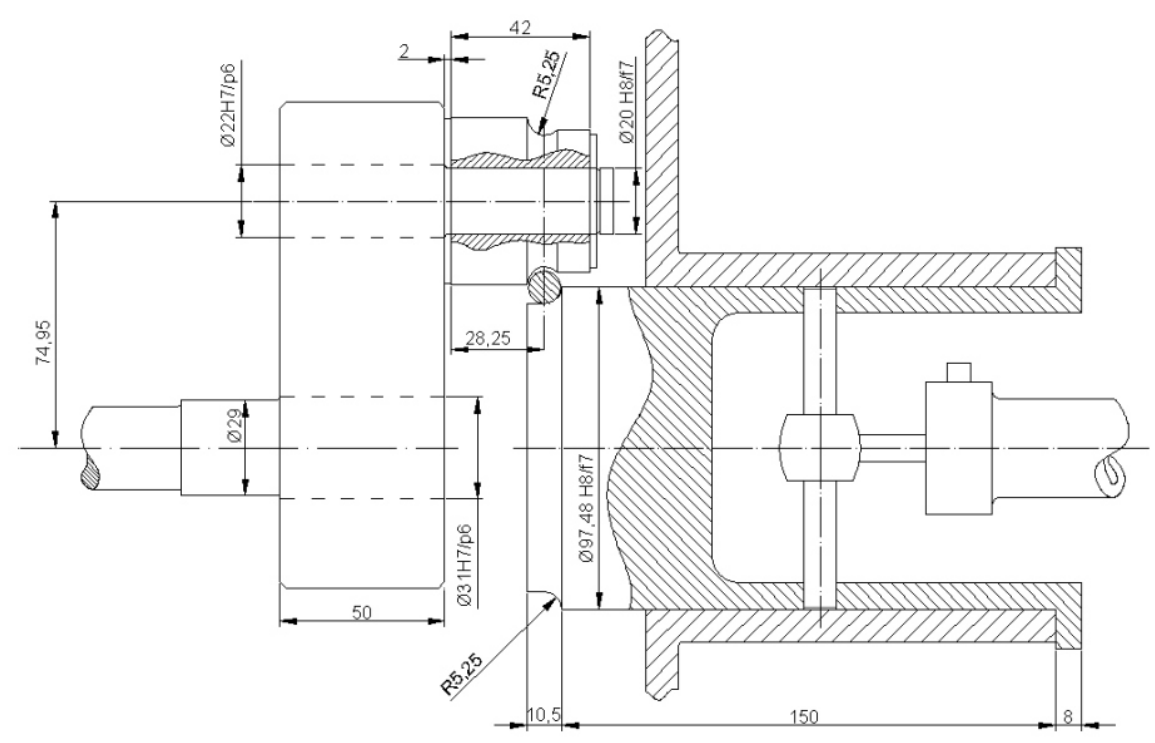

Figura 9. Rediseño para el conjunto palanca y rodillo seguidor de la máquina dobladora.

La condición de rigidez del conjunto palanca y rodillo seguidor resulta más crítica que la resistencia a la fatiga volumétrica, siempre que sean empleadas buenas prácticas de diseño con geometrías que eviten la elevación local de las tensiones.

No fue posible garantizar en el rediseño el no aplastamiento de la barra debido a las tensiones de contacto. Pero esto sólo es limitante cuando se tienen restricciones estrechas de tolerancia de forma para la sección transversal de la barra.

\section{Referencias bibliográficas}

Bhatti, M. A. (2000). Practical optimization methods with Mathematica applications. New York: Springer-Verlag.

Feodosiev, V. I. (1985). Resistencia de materiales. Moscú: Editorial MIR.

Groover, M. P. (1997). Fundamentos de manufactura moderna. Prentice-Hall Hispanoamericana, S.A.

Mielnik, E. M. (1991). Metalworking science and engineering. New York: McGraw-Hill, Inc.
Norton, R. L. (1998). Machine design. New Jersey: Prentice Hall.

Ullman, D. G. (1992). The mechanical design process. New York: McGraw Hill, Inc. 